\title{
Ciéntifica
}

http://revistas.udistrital.edu.co/ojs/index.php/revcie/index

CIDC

Artículo De REFLEXIÓN

\section{Transformación del método cartesiano en las ediciones de la geometría de Bernard Lamy}

\author{
Transformation of the cartesian method in the editions of the Geometry of Bernard Lamy
}

Transformação do método cartesiano nas edições da Geometria de Bernard Lamy

Jhon Helver Bello-Chávez' ${ }^{\circledR}$

Recibido: agosto 2019

Aceptado: abril 2020

Para citar este artículo: Bello-Chávez, J. H. (2020). Transformación del método cartesiano en las ediciones de la geometría de Bernard Lamy. Revista Científica, 38(2), 216-228. https://doi.org/10.14483/23448350.14580

\section{Resumen}

Este artículo presenta una contribución a la historia de la enseñanza del álgebra, el estudio del método cartesiano de resolución de problemas geométricos en las diferentes ediciones del libro Les Élémens de Géométrie (Lamy, 1685). Este libro de texto circuló en Francia durante casi un siglo, se usó en los colegios de la congregación de los oratorianos y estableció un modelo de enseñanza de la geometría que desarrolló el análisis propuesto en la Géométrie (Descartes, 1637). Al analizar la sección sobre el método en las ediciones del libro desde 1685 hasta 1756 se evidenció que la enseñanza se concentró en desarrollar una técnica por medio de reglas para usar el análisis. En cada edición la importancia de los diagramas en la aplicación del método fue disminuyendo y la construcción de la solución de ecuaciones de primer y segundo grado fue perdiendo importancia. Como cartesiano, Lamy recurrió al principio de simplicidad de esta filosofía y del método, por lo tanto, usó relaciones entre líneas descritas por medio de la proporcionalidad y la semejanza de triángulos como medio para organizar sus justificaciones.
Palabras clave: historia de la matemática, enseñanza del álgebra, educación siglo XVIII, resolución de problemas, educación matemática.

\begin{abstract}
This article presents a contribution to the history of the teaching of algebra, the study of the Cartesian method of solving geometric problems in the different editions of the book Les Élémens de Géométrie (Lamy, 1685). This textbook circulated in France for almost a century, was used in colleges of the congregation of the Oratorians and established a teaching model of geometry that developed the analysis proposed in the Géométrie (Descartes, 1637). When analyzing the section on the method in the editions of the book from 1685 to 1756 it was evident that the teaching focused on developing a technique by means of rules to use the analysis. In each edition the importance of the diagrams in the application of the method was decreasing and the construction of the solution of equations of first and second degree was losing importance. As a Cartesian, Lamy resorted to the simplicity
\end{abstract}

1. Universidad Distrital Francisco José de Caldas. Bogotá, Colombia. jhbelloc@udistrital.edu.co 
principle of this philosophy and method, therefore, he used relationships between lines described by means of proportionality and the similarity of triangles as a means to organize their justifications.

Keywords: history of mathematics, teaching of algebra, education XVIII century, problem solving, mathematics education.

\section{Resumo}

Este trabalho apresenta uma contribuição para a história do ensino de álgebra, o estudo do método cartesiano de resolver problemas geométricos nas diferentes edições do livro Les Élémens de Géométrie (Lamy, 1685). Este livro circulou na França por quase um século, foi usado nas escolas da Congregação do Oratório e estabeleceu um modelo de ensino da geometria que se desenvolveu a análise proposta Géométrie (Descartes, 1637). Ao analisar a seção sobre o método nas edições do livro de 1685 a 1756, ficou evidente que o ensino se concentrava em desenvolver uma técnica por meio de regras para usar a análise. Em cada edição a importância dos diagramas na aplicação do método foi diminuindo e a construção da solução de equações de primeiro e segundo grau foi perdendo importância. Como cartesiano Lamy virou o princípio de simplicidade desta filosofia e método, portanto, ele utilizado relações entre as linhas descritas pela proporcionalidade e triângulos semelhantes, como um meio para organizar as suas justificações.

Palavras-chaves: história da matemática, ensino de álgebra, educação do século XVIII, resolução de problemas, educação matemática.

\section{Introducción}

La divulgación de un saber matemático por medio del sistema educativo requiere una adecuación del conocimiento para la enseñanza. Nos interesa el caso del libro de texto de Lamy por ser uno de los primeros en proponer para la educación las ideas cartesianas; específicamente el método de resolución de problemas geométricos para la enseñanza de la geometría.

En la actualidad se piensa que el método contribuye con la conformación del sistema simbólico de las matemáticas y se le atribuye al trabajo de Descartes este horizonte (Stacey et al., 2004; Sutherland et al., 2002). Sin embargo, los análisis históricos de la obra muestran la naturaleza geométrica de los símbolos. Estos existieron para referirse a relaciones y objetos geométricos.

Lamy fue un ferviente seguidor de las ideas cartesianas. Dentro de su obra trabajó para la enseñanza una interpretación del método para resolver problemas geométricos. Descartes, en la regla $\mathrm{V}$, describe el método de la siguiente manera:

Todo el método consiste en el orden y disposición de aquellas cosas a las que se ha de dirigir la mirada de la mente a fin de que descubramos alguna verdad. Y la observaremos exactamente si reducimos gradualmente las proposiciones complicadas y oscuras a otras más simples, y si después intentamos ascender por los mismos grados desde la intuición de las más simples hasta el conocimiento de todas las demás. (Descartes, 2010, p. 93)

En el caso de las matemáticas, se consolidó a través del método de descubrimiento llamado por Pappus: análisis. Lo enunció así:

$\mathrm{Si}$, pues, deseamos resolver un problema, inicialmente debe suponerse efectuada la resolución, dando nombre a todas las líneas que se estimen necesarias para su construcción, tanto a las que son desconocidas como a las que son conocidas. A continuación, sin establecer distinción entre líneas conocidas y desconocidas, debemos descifrar el problema siguiendo el orden que muestre, de modo más natural, las relaciones entre estas líneas, hasta que se identifique un medio de expresar una misma cantidad de dos formas [...]. (Descartes, 1996, p. 392)

El uso del método al nombrar las líneas y sus relaciones supuso la existencia de una solución a través de un diagrama. Es decir, la técnica partió de la construcción de una herramienta dentro de la práctica específica de la geometría cartesiana, lo 
que permitió externalizar la información del problema en un espacio de trabajo en donde se suscitó la inferencia.

El contexto de estos diagramas fue una nueva teoría de magnitudes que se desarrolló a partir de interpretar las operaciones de suma, sustracción, producto, división y extracción de raíz cuadrada utilizando relaciones entre líneas. Esta estructura se convirtió en el escenario que permitió interpretar los problemas desde una nueva metafísica, en la cual los objetos están definidos a partir de relaciones (Macbeth, 2004).

Parte del proceso se llevó a cabo en la conformación y análisis del diagrama, luego las relaciones eran expresadas algebraicamente. Descartes lo afirma al inicio de la Geometría: "Todos los problemas de la geometría pueden ser deducidos fácilmente a términos tales que no sea necesario posteriormente para construirlos, sino conocer la longitud de algunas líneas" (1996, p. 389).

Después de obtener la expresión simbólica que representa las relaciones que posibilitaban la solución del problema, el método continuó con la construcción de la solución de una ecuación. Descartes condicionó la aceptabilidad de una curva como geométrica a los medios de construcción con los que fue construida (Bos, 2001). En Geometría se superó las limitaciones de la regla y el compás mediante la aceptación de otros instrumentos para validar la existencia de una curva. El resultado es otro diagrama que mostró las relaciones entre segmentos o curvas que permitieron definir cada punto de una nueva curva y obtener el conjunto de puntos que la define. Todas las ecuaciones que cumplían una característica se identificaron en una clase curva, de primer grado, segundo..., etc.

\section{El material de estudio}

Todas las ediciones del texto Les élémens de Géométrie ou de la mesure du corps de Lamy tuvieron su contenido distribuido entre varios libros y al interior de cada uno por capítulos. Este estudio analizó en siete impresiones el apartado dedicado al método. Las impresiones fueron organizadas en tres grupos de ediciones, en cada grupo el contenido del libro es el mismo, pero entre estos existen modificaciones en el texto. Para el grupo 1: impresiones de 1685 y 1692, se analizó el libro V; para el grupo 2: ediciones de 1710, 1732, 1734 y 1740, y el grupo tres la edición de 1758, el libro VI.

Siguiendo el método propuesto para el análisis de libros de texto antiguos de Schubring (1987), se revisó la práctica matemática propuesta por Lamy en cada grupo de ediciones. Se develó la transformación que hubo para la enseñanza de la geometría analizando la definición de método, su cambio en relación con el propósito de desarrollo de las ideas cartesianas, la evolución en el uso de los diagramas y en el tratamiento de las proporciones.

\section{Análisis sobre el método. Primer grupo}

El método para la solución de problemas propuesto por Lamy en el capítulo $\mathrm{V}$ se describe a través de las siguientes ocho reglas (1685, pp. 282-289):

- Como todo no es desconocido en una pregunta, representar lo conocido y suponer lo desconocido.

- $\quad$ i se propone una pregunta debemos saber elegir ciertas cantidades y en consecuencia comprender si la pregunta es determinada o indeterminada.

- Se debe considerar cuidadosamente qué buscar en una pregunta, para indicar lo que se conoce y para distinguir lo que no. Las cantidades conocidas se indican con las primeras letras del alfabeto y las desconocidas con las últimas.

- En una pregunta se descubre lo que relaciona una magnitud desconocida por las propiedades de la figura que se aprovecha para resolver esta cuestión y por las relaciones que las cantidades desconocidas tienen con las conocidas.

- Examinar las cantidades y expresar lo desconocido de dos maneras se llama ecuación. 
- Los términos que representan una pregunta deben expresarse de modo que si es posible solo hay una letra desconocida.

- Realizar procedimientos para igualar las cantidades desconocidas.

- Es necesario reducir los términos de una pregunta a los términos más simples.

Estas reglas relataron exhaustivamente las actividades que configuraron el método y que permitieron llevar a cabo la resolución de los problemas. Cada regla era un paso en la conformación de la resolución. Lamy interpretó el método a través de una técnica que consistió en pasar del análisis de la pregunta a la formulación de una ecuación que la representa, mediante el desarrollo de un diagrama.

En los cuatro capítulos que anteceden a la formulación de las reglas se dedicó a mostrar la importancia de analizar la pregunta por medio de una figura, que es un diagrama. Expresó que esta forma de entender una pregunta permitía evidenciar las relaciones que solucionan la situación. Por ejemplo:

El área de un triángulo es igual a la mitad de la suma de sus tres lados multiplicados por el radio de un círculo que se inserta en él.

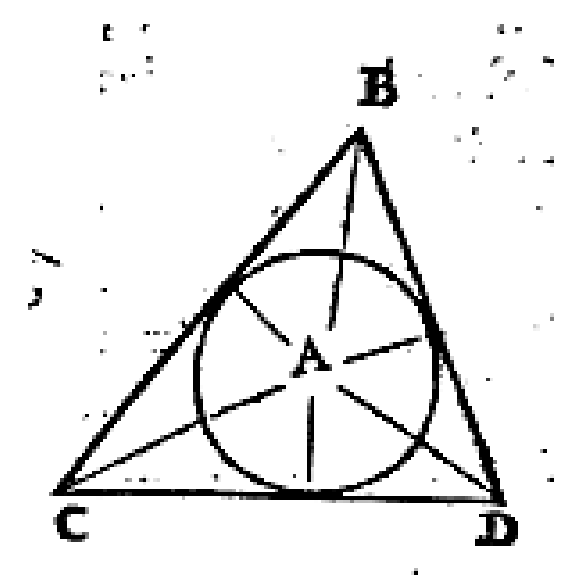

Figura 1. Problema círculo inscrito en triángulo.

Fuente: Lamy (1685, p. 269).

Lamy afirmó que al apreciar la figura se evidencia que la propiedad que enuncia el problema es verdadera y se puede garantizar por medio del diagrama. Como se observa en la figura 1 se forman tres triángulos cuyas bases son cada uno de los lados del triángulo CBD y con altura igual al radio del círculo inscrito. El triángulo $\mathrm{CBD}$ queda compuesto por los triángulos $\mathrm{CAB}, \mathrm{BAD}$ y $\mathrm{CAD}$. El diagrama es fundamental para inferir la solución.

Coincidiendo con el método propuesto por Descartes, el diagrama inicial del problema entrega las relaciones que se necesitan para suponer la solución. Este es el primer paso en el proceso de análisis, el cual en este grupo de ediciones es amplio en explicaciones y ejemplificaciones. La propuesta de análisis de la pregunta y la conformación de una figura que permita evidenciar las relaciones del problema está acompañada por una heurística alrededor de la descomposición en triángulos (Barbin, 2010). Esta técnica, que aparece en el libro II de los Elementos de Euclides, se sugirió en el transcurso del texto para la resolución y compresión de todos los problemas. En este aspecto recae la simplicidad que proporcionó el método, reducir los problemas a triángulos fue la manera de establecer las relaciones más simples, un principio de relación entre segmentos, una proporción por medio del teorema de Tales.

En el capítulo IV describe cuatro medios para analizar la figura con el fin de encontrar las relaciones que permitan resolver una pregunta, a saber: el conocimiento de las propiedades de los objetos involucrados, de los ángulos, de las razones y proporciones, y reducir las figuras a triángulos semejantes (Lamy, 1685, p. 274).

Lamy antes de presentar las reglas enseñó dónde encontrar las relaciones que posibilitan solucionar un problema. El estudio del diagrama implicó comprender la posibilidad de establecer representaciones simbólicas para las relaciones que se establecían o inferían a partir de la solución. Por ejemplo, en la siguiente situación:

El producto o el rectángulo que hacen las diagonales $A C$ y $B D$ es igual a la suma de los rectángulos $B C$ por $A D$ y $A B$ por $D C$. Lados opuestos del cuadrilátero $A B C D$ que está inscrito en una circunferencia. 


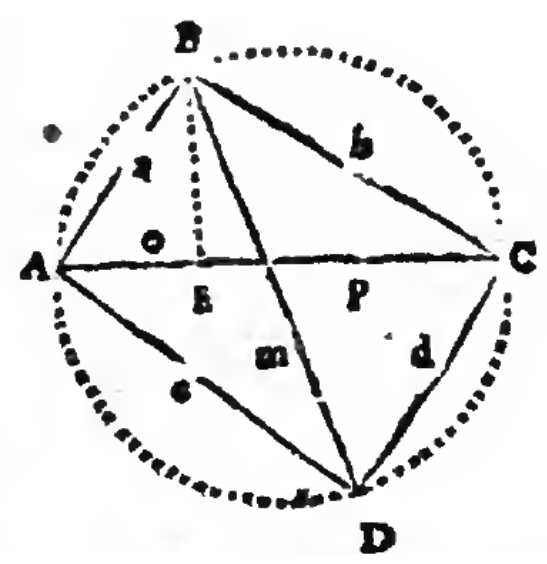

Figura 2. Cuadrilátero inscrito.

Fuente: Lamy (1685, p. 274).

Después de asignarle una letra a los segmentos de recta, Lamy propone una línea auxiliar $B E$, con la característica que los ángulos $A B E$ y $D B C$ sean iguales. De esta relación deduce las siguientes proporciones $\frac{B D}{A D}=\frac{B C}{C E} ; \frac{m}{c}=\frac{b}{p}$.

A continuación, evidencia que consecuencia de la condición de $B E$, los triángulos $B D C, B A E$ son semejantes $y$, por tanto, es posible plantear varias proporciones para llegar al resultado. Una muestra del estudio de la figura por medio del uso de los medios propuestos; el diagrama al igual que en la obra de Descartes se convierte en una herramienta de exploración de la solución.

Finalmente, muestra que la mejor solución de un problema es cuando se obtiene por medio del método una expresión para la generalización de la situación. Algunos ejemplos se plantearon al final del libro:

Sea $\mathrm{BAD}$ un triángulo isósceles, proponemos cortar sus lados $\mathrm{AB}, \mathrm{AC}$ por una paralela a la base $\mathrm{BC}$; de modo que esta paralela sea igual a lo que queda de los lados; es decir, que $\mathrm{DB}=\mathrm{DE}$

\section{Primera solución}

Supongo que está hecho. Que $B D=D E$, entonces el triángulo $B D E$ es isósceles y los ángulos $D B E$ y $D E B$ son iguales. Los ángulos $C B E$ y $E B D$ son iguales, dejando que $E B C$ y $E B D$ sean iguales. Por lo tanto, la línea $B E$ corta la mitad del ángulo $D B C$. De donde se deduce que son triángulos isósceles, como $B A C$. Por esta propiedad del triángulo isósceles, encuentro este medio para resolver el problema propuesto (Lamy, 1685, p. 314).

\section{Segunda solución}

Supongo el problema resuelto, nombro a $A B$ que es conocido $a$ y $d$ a la base $B C$, también conocido. Llamo $x$ al tamaño desconocido $A E$; así que $E C=a-x$, también $D E=a-x$ entonces $a: d:: x: a-x$ obtenemos $a a-a x=d x$. Agrego a ambos lados $a x$, entonces $a a=d x+a x$. Suponiendo que $c=d+a$ entonces $c x=d x+a x$ y por lo tanto, en lugar de $d x+a x$ coloco $c x$, tengo $a a=c x$; dividiendo la ecuación por $c$ tengo por consecuencia, $\stackrel{-}{-} c$, $a, x$ Entonces solo se trata de agregar un tercio proporcional a dos líneas conocidas, que son los primeros dos términos de una progresión. La línea $A B$ unida con la línea $B C$ es el primer término y $A B$ el segundo (Lamy, 1685. p. 314).

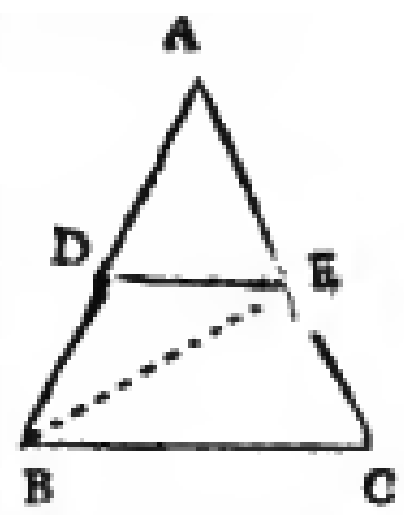

Figura 3. Problema con dos soluciones.

Fuente: Lamy (1685, p. 314).

En las dos formas de resolver el problema usó pasos del método, sin embargo, optó por recomendar la segunda solución; pues indicó que es una forma analítica general y no es particular del problema. Se puede aplicar este mismo razonamiento en la solución de todo triángulo isósceles. El análisis permitió establecer la ecuación que representa esta solución. 


\section{Análisis sobre las proporciones. Primer grupo}

Lamy afirma que por las características educativas de la obra va a abordar los problemas lineales y planos. Es decir, problemas cuya solución requiere una ecuación de primer o segundo grado. Solo nombró la posibilidad de existencia de otros tipos de ecuaciones; por ejemplo, planteó la ecuación de tercer grado que resuelve el problema de la trisección del ángulo.

La construcción para las ecuaciones de grado uno y dos los trabajó a partir de la media y tercera proporcional. Mostró la correspondencia entre la representación simbólica y las relaciones más básicas de la matemática. De esta manera, aplicó el método llevando la solución a un principio de la geometría.

Para el caso de las ecuaciones de segundo grado presentó la siguiente construcción:

Sea $y y=b b+y d$ encuentre el valor de y. Levanto en una de las extremidades de $b$ una perpendicular igual a la mitad de $d$. Desde la parte superior de esta perpendicular como centro, hago un círculo y por su centro conduzco una secante hasta $D$. (Lamy, 1685, p. 298)

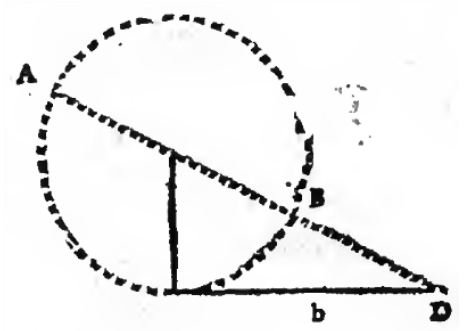

Figura 4. Construcción ecuaciones de segundo grado.

Fuente: Lamy (1685, p. 299).

La línea $b$ es una tangente por construcción, y dado que el radio del círculo es igual a la mitad de $d$, el diámetro entero $A B=d$, llamo $y$ a la secante $A D$, y $x$ la parte $D B$ que está fuera del círculo. Dado que el todo multiplicado por las partes es el producto del todo por el todo, $y y=y d+y x$,
: $y, b, x$ entonces $y x=b b$ sustituyendo así en la ecuación anterior $y y=y d+y x$ el cuadrado $b b$ en lugar de $y x$, se obtiene $y y=b b+y d$; haciendo lo que hicimos encontramos la línea $A D$, que es igual a la magnitud, previamente desconocida (Lamy, 1685, p. 299).

En la presentación de Lamy existen cambios significativos respecto al tratamiento realizado por Descartes en 1637. Se usa la misma construcción y resulta el mismo diagrama, pero los argumentos son diferentes a los originales. En Descartes se llega a que $z=\frac{1}{2} a+\sqrt{\frac{1}{4} a^{2}+b^{2}}$ por medio del teorema de Pitágoras, haciendo uso de la siguiente construcción:

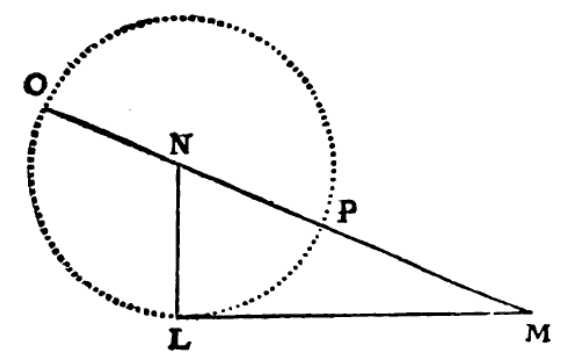

Figura 5. Construcción ecuaciones de segundo grado.

Fuente: Descartes (1996, p. 394).

Construyo el triángulo rectángulo $N L M$, cuyo lado $L M$ es igual a $b$ (raíz cuadrada de la cantidad desconocida $b b$ ) y el otro lado $L N$ es igual a $\frac{1}{2} a$, esto es: la mitad de la otra cantidad conocida que estaba multiplicada por $z$, la cual he supuesto que es la línea desconocida. Seguidamente prolongando $M N$, que es la base de este triángulo, hasta $O$, de suerte que $N O$ sea igual a $N L$, la línea $O M$ es $z$, la línea buscada. (Descartes, 1996, p. 394)

Usando el teorema de Pitágoras Descartes concluye que la hipotenusa del triángulo es:

por lo tanto,

$$
N M=\sqrt{\frac{1}{4} a a+b b}
$$

$$
z=\frac{1}{2} a+\sqrt{\frac{1}{4} a a+b b}
$$


Esta diferencia en la argumentación de la solución de las ecuaciones de segundo grado entre Lamy y Descartes permite comprender que para Lamy la tercera proporcional se convirtió en una forma simple de relación matemática, la cual permitía llegar a una generalización del tratamiento aritmético sin construcción. Por ejemplo, en la descripción del capítulo IX enuncia: "las ecuaciones bidimensionales se pueden reducir a una progresión de tres términos, lo que proporciona una forma más natural de resolver problemas planos" (1685, p. 301).

Son las proporciones y en especial la tercera proporcional, la idea simple que permite generalizar el procedimiento para hallar la raíz de la ecuación. Lamy muestra como a partir del método, del análisis, se establece el principio fundamental que sustenta la resolución, la relación básica que permite la existencia y justificación de una idea matemática: "[...] al buscar por análisis la resolución de un problema, después de llegar a una de las tres ecuaciones anteriores, debe reducirse a una progresión de tres términos, en la que siempre se conoce la media proporcional [...]" (1685, p. 305).

Los elementos que justificaron este tipo de deducciones fueron las proporciones. En consecuencia, algunas "fórmulas" para la solución de ecuaciones van desplazando la construcción geométrica. Para el caso de las ecuaciones de segundo grado, trabajó los siguientes casos:

- La ecuación $x x=a x+b b$ se reduce " $x-a, b, x$ porque $x x-a x=b b$, al sumar $a x$ en ambos lados, se tiene la ecuación en cuestión $x x=a x+b b$.

- La ecuación $x x=a x-b b$ se reduce : $x, b, a-x$. Como $a x-x x=b b$ al sumar $x x$ en ambos lados, se tiene $a x=b b+x x$ , eliminando $b b$ en ambos lados, se tiene $a x-b b=x x$.

- La ecuación $x x=b b-a x$ se reduce a " $x+a, b, x$ porque $x x+a x=b b$, eliminando en cada lado, tenemos $x x=b b-a x$.
Estas tres maneras de expresar las ecuaciones median entre la construcción de la solución de la ecuación y la representación simbólica. La pretensión de Lamy de hacer conocer las cosas de la manera más natural termina siendo reducir los problemas que trabaja a un principio elemental para las matemáticas, como lo es la tercera y la cuarta proporcional. Este proceso mediador y generalizador es más aritmético que geométrico.

\section{Cambios en relación con el método. Segundo grupo de ediciones}

Este grupo de ediciones va desde 1710 hasta la impresión de 1740. Del prefacio se deduce que fue revisada y ampliada por el mismo Lamy. Se adicionó un libro sobre las propiedades que cumple cualquier magnitud y al final del texto un apartado sobre secciones cónicas. En la introducción indicó que el libro Vı lo dedicaba al método, lo que los geómetras Ilamaban análisis y que esta forma de proceder contribuye a formar mentes más exactas y penetrantes.

En este libro anunció la diferencia en el abordaje de los problemas con los libros anteriores "el método que hemos seguido hasta ahora ha sido considerar la idea de las cosas de las que hablamos y luego derivar sus propiedades [...] este método supone lo conocido" (Lamy, 1710, pp. 374-375). Enuncia que durante el capítulo va a trabajar con un nuevo método: "hay otro método con el que uno encuentra lo que no sabe [...] por eso se llama método de invención, mientras al primero se puede llamar método de doctrina" (Lamy, 1710, p. 375).

Tres asuntos Ilaman la atención en el tratamiento de problemas con el método, el primero, la distribución de las reglas ${ }^{2}$ durante el libro, se adecuó un capítulo para explicar de cada una. Segundo, se perdió el énfasis en la elaboración del diagrama, por lo menos en términos de extensión

2. El autor no habla explícitamente de reglas; sin embargo, los enunciados tienen la misma intención y práctica que en la primera edición. 
y ejemplificación en el texto. En algunos problemas al tener que resolver la ecuación solución del problema, se recurre al diagrama de otro problema o a uno general. Tercero, en esta edición no se ve un énfasis en la heurística de descomposición de triángulos.

Las seis reglas que se deducen de las descripciones de los capítulos son más específicas en la actividad que se debe realizar:

- Es necesario concebir y expresar claramente la pregunta por medio de una figura.

- Podemos expresar las líneas de todas las magnitudes de las que hablamos en una pregunta y hacer sobre ellas todas las operaciones de aritmética, sin conocerlas todas.

- Distinguir lo conocido de lo desconocido, para considerar si el problema está determinado o es indeterminado y distinguir entre tres cosas; las líneas que se conocen, aquellas líneas que no se conocen y todas las relaciones entre líneas conocidas y desconocidas.

- El conocimiento de las relaciones que están entre las líneas que conforman la figura de un problema da la forma de igualar o encontrar expresiones dobles de la misma magnitud.

- Encontrar tantas ecuaciones como líneas desconocidas y reducir todas estas ecuaciones a una.

- Reducir los términos de una ecuación a la expresión más simple y luego dejar la magnitud desconocida en uno de los lados de la ecuación.

En general el método es más técnico, más instructivo que el anterior. Se dedica mayor espacio a explicar cómo reducir ecuaciones, cómo expresar una misma magnitud a partir de una expresión simbólica diferente y en hacer operaciones de términos semejantes. Estos aspectos fortalecen la necesidad de conocimiento de aspectos de los libros anteriores. Por ejemplo, en el capítulo IV plantea:

Lo que hemos visto en el tercer libro sobre las razones, también es una fuerte de ecuaciones diferentes; si $a+b=x$, entonces $a a+2 a b+b b=x x$. Si $x$ es la hipotenusa de un triángulo rectángulo y los lados son $a$ y $b$, entonces $a a+b b=x x$ así $x x-a a=b b \quad$ o $x x-b b=a a$. (Lamy, 1710, p. 386)

En esta edición recurrió a propiedades algebraicas, a la relación entre la multiplicación y la cuarta proporcional y en general a aspectos que permitieron ir generalizando ecuaciones, eso hace que las explicaciones sobre las reglas fueran más operativas que en la versión anterior.

\section{Cambios en relación con las proporciones}

En los primeros planteamientos de esta edición Lamy propone las relaciones entre proporción y la estructura de la magnitud. Por ejemplo, al plantear la multiplicación lo hace en los términos planteados por Descartes y luego dice:

[...] por lo tanto el producto de tres letras, como $a b c$, puede establecer estas dos proporciones $1: c:: a b: a b c$ y $1: a b:: c: a b c$, esto muestra que el producto de cuatro líneas $a b c d$ establece tres proporciones; el producto de cinco líneas abcde cuatro proporciones y en todas estas proporciones el producto final es una línea. (Lamy, 1710, p. 380)

Describió una generalización en el producto, para lo cual no usó construcciones de las ecuaciones. Al mostrar las posibles proporciones, Lamy inicia un proceso de separación entre la operación entre segmentos y su construcción. Ese estilo es frecuente en esta edición. El último capítulo lo dedicó a resolver algunos problemas; las ecuaciones producto del tratamiento de proporciones y la solución de ecuaciones por medio de la tercera proporcional son las técnicas usadas. Consideró soluciones en donde no se presenta ni el diagrama del problema, ni la construcción de la ecuación. Por ejemplo:

Encontrar cada lado del triángulo $\mathrm{ABC}$, conociendo la suma de cada uno de sus lados. 


\begin{abstract}
Si $A B=x$ y $A B+B C=a$ y $A B+A C=b$ y $\quad B C+A C=c, \quad$ entonces $\quad B C=a-x$ y $\quad A C=b-x \quad$ y $\quad A C+B C=c$, entonces $a-x+b-x$ о $a+b-2 x=c$. Sumando $2 x$ en ambos lados, tendremos $a+b=c+2 x$, estando $c$ en ambos lados tendremos $a+b-2 x=c$ . Entonces para encontrar el valor de $x$, tenemos que unir las dos líneas $a$ y $b$ para restar la línea $c$, la mitad de lo que quede será el valor de $x$. (Lamy, 1710, p. 405)
\end{abstract}

Para el caso de las ecuaciones de segundo grado se hace referencia a realizar la construcción que determina la tercera proporcional.

Considere esta progresión de tres líneas $x+d, b, x$ o $x-d, b, x$. Se conoce el promedio $b$, y la diferencia $d$ entre los extremos $x+d$ y $x$, halle el valor de $x$

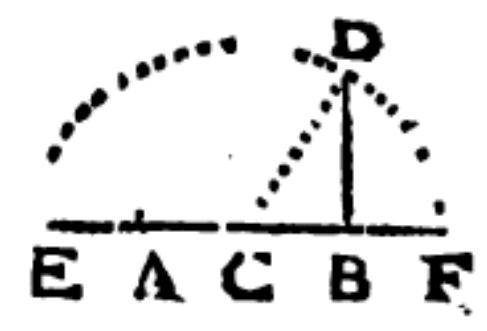

Figura 6. Solución de ecuaciones de segundo grado por progresiones.

Fuentes: Lamy (1710, p. 304).

Sea $A B$ igual a $d$, y en $B$, elevo perpendicularmente $B D$ igual a $b$. Luego $C$ es la mitad de $A B$. Con el intervalo $C D$ hago un círculo. Prolongo $A B$ hasta el círculo. Después $A E$ o $B F=x$, porque $=x+d, b, x$. Si la progresión es " $x-d, b, x$ debemos hacer lo mismo, pero en este caso donde $x=E B$, el término más pequeño es $B F$ igual a $E B-A B$ o $x-d$. (Lamy, 1710, p. 304)

En conclusión, la distribución por capítulos para cada una de regla permitió un mejor detalle de las reglas algebraicas involucradas. Se eliminó el énfasis en el diagrama de la primera edición y se fortaleció la generalización de procedimientos para la solución de problemas. La tercera y cuarta proporcional se convirtieron en una forma de razonamiento para la solución de varios problemas por medio de la misma construcción; por tanto, la relación entre álgebra y geometría quedó encapsulada por el razonamiento proporcional.

\section{Cambios respecto al método. Tercer grupo}

La séptima edición del texto data de 1758, momento para el cual Lamy ya había fallecido. No es posible saber el nombre de sus editores; se deduce por la autorización que manifiesta la congregación que las adiciones y alteraciones al texto fueron realizadas por personas de esta organización. Específicamente, en el aviso a las bibliotecas siempre se refieren a nosotros en términos de la comunidad. Para el libro VI sobre el método y el tratado de secciones cónicas se refieren a uno de nosotros, un oratoriano que se comprometió a realizar estas adecuaciones.

En este mismo apartado se anuncia que el capítulo VI es un curso sobre análisis y síntesis en problemas que son solucionables con ecuaciones de primer, segundo y tercer grado. De igual manera, anuncian que el libro sobre secciones cónicas fue modificado, de tal manera que incluye todas las proposiciones esenciales para estudiar la física de Newton. La física matemática fue un objetivo de la educación universitaria en Francia entre 1740 y 1770 (Belhoste, 1993, pp. 2 y 3).

Respecto a las ediciones anteriores las modificaciones al libro VI se leen desde el inicio del texto, ahora el método tiene nombre, analítico y sintético. Se aclara que su uso es solucionar problemas que presentan una aplicación del álgebra a la geometría.

En esta edición se reconoce que el análisis busca un principio, una proposición fundamental que determine la veracidad sobre el supuesto mismo, aspecto que se comprueba por la síntesis. Aunque se describen dos métodos por separado, Lamy reconoce interacción entre estas dos formas, hablando sobre los métodos: “[...] no son 
tan incompatibles, pueden estar juntos, la mayoría de las veces se ayudan mutuamente" (Lamy, 1758, p. 515).

Relacionó el método analítico con una buena enseñanza; afirmó que los pasos del método son los aspectos que un maestro debe privilegiar en vez de "el conocimiento seco de los teoremas de la geometría" (Lamy, 1758, p. 516). Mostró su oposición a la enseñanza desde la estructura del texto de Euclides. Esta discusión sobre lo analítico y lo sintético en la enseñanza seguirá hasta después de la Revolución francesa, momento en el cual grupos de filósofos y pedagogos apostaron por el método analítico y el lenguaje algebraico. Sin embargo, años más tarde se daría un restablecimiento del método sintético (Schubring, 2005, pp. 291-293).

En esta edición el autor reconoce al álgebra como un instrumento que posibilita un buen análisis: "podemos estar convencidos de que el álgebra aplicada a la geometría es el álgebra sujeta a las reglas del análisis, en la medida en que el objeto es el descubrimiento de las propiedades de las líneas [...]" (Lamy, 1758, p. 519).

En este sentido, los objetivos del texto cambian, el conocimiento sobre el método se instrumentó por medio del álgebra. Como afirma el autor, es suficiente que la mano desarrolle los cálculos para liberar a lo desconocido.

En esta edición las reglas del método son cinco y están explicadas en el primer capítulo:

- Tener conocimiento previo que nos ayude a comprender la naturaleza de lo desconocido.

- Las condiciones establecidas en el problema deben ser discutidas cuidadosamente.

- Discutir las relaciones entre lo conocido y lo desconocido, son estas expresiones los instrumentos de análisis. Por lo tanto, debe prestar toda la atención a: eliminar de la pregunta todas las ideas que no conducen a la verdad. Examinar la pregunta en todas sus partes, una después de la otra en orden natural, comenzando por la más simple, es decir, por las que tiene menos relaciones. Recomponer las relaciones de acuerdo con las condiciones más complicadas del problema y si no es posible hacer las relaciones comparado las condiciones, recurra a una idea intermedia que finalice con dos relaciones para asignar su relación de mediación.

- Abreviar las relaciones, expresarlas de la manera más simple posible, ordenarlas y con signos reconocibles.

- Cuando se comprenden y simplifican las relaciones de lo conocido con lo desconocido, formamos ecuaciones o proporciones.

En estas reglas se enfatiza en hacer operativas las relaciones que se determinan a partir de reflexionar sobre el problema. Este aspecto se confirma en los capítulos II y III, en los que se trabajan las propiedades y operaciones de las ecuaciones.

Sin embargo, en la sección de problemas las situaciones se plantearon a través de diagramas, los cuales no están inmersos en el texto como en los libros anteriores, las construcciones están en la parte final del libro, como un anexo. Esto solo sucede en el libro VI y en el tratado de secciones cónicas. En estos libros se separó la construcción de la ecuación de la solución del problema. El autor afirma en dos ocasiones que las construcciones son más elegantes que el trabajo algebraico, pero este último permite hallar la solución de manera más ágil. Por ejemplo:

Conociendo las diferencias en ambos lados de un triángulo rectángulo con la hipotenusa, encuentre los tres lados de este triángulo.

Deje $x$ ser la hipotenusa, $a$ el exceso con el lado más largo, $b$ el exceso de la hipotenusa con el lado más pequeño. Entonces, el lado más grande $x-a, \mathrm{y}$ el más pequeño $x-b$. La condición de ángulo recto da $x x-2 a x+a a+x x-2 b x+b b=x x$ se reduce a $x x-2 b x-2 a x=-a a-b b$ de donde tenemos la ecuación $x=a+b \pm \sqrt{2 a b}$. (Lamy, 1758, p. 546) 
Para encontrar la solución a la ecuación, se presenta la siguiente construcción:

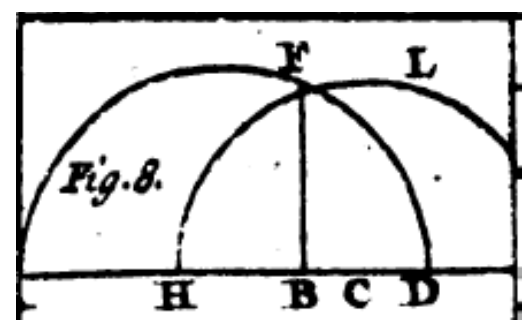

Figura 7. Construcción de ecuaciones.

Fuente: Lamy (1758, p. 637).

Tome una línea $A B=b$ y en esta línea $B C=a$, extendemos la línea $B C$ a $G$ y tomamos $C D=B C$; en $A D$, describimos un semicírculo $A F D$, en el punto $B$, se eleva una recta $B F$ y siguiendo al punto $C$ como centro y con $B F$ para el radio, se describe un nuevo semicírculo $H L G$, y las líneas $A G, A H$ serán las raíces de la ecuación. (Lamy, 1758, p. 546)

La construcción geométrica es usada para expresiones como la del problema anterior, en el caso de ecuaciones que están clasificadas como de primer y segundo grado, el texto refiere a la facilidad de la construcción.

\section{Cambios en relación con las proporciones. Tercer grupo}

En esta edición se afirmó que las propiedades que se cumplen en la aritmética también se cumplen para la geometría. En el caso de la multiplicación indicó que la operación se reduce a encontrar una tercera línea recta que es el producto bajo una unidad, este hecho se justifica - como en Descartes - por medio de una cuarta proporcional.

Se precisaron combinaciones de las reglas de las operaciones, suma, resta, producto, división y raíz cuadrada. En este sentido, se indica que es posible construir fracciones como por ejemplo,

$$
\sqrt{\frac{a^{3} b c}{f g^{2}}}
$$

el procedimiento que presentó y que consideran el más fácil:
Sea primero $f: a:: b \quad \frac{a b}{f}=d$. La fracción, por sustitución de esta línea se convertirá en $\frac{a^{2} d c}{g^{2}}$, busco de nuevo un prca $a^{2} h^{2}$ io proporcional entre $d$ y $c$ que llamo b que da $\overline{g^{2}}$ desde donde vemos, haciendo las raíces, solo para tener el valor de esta fracción y desde ahí el de la primera, que es igual, solo hay que encontrar un cuarto proporcional a las líneas $g$, $a$ y $b$. (Lamy, 1758, p. 534)

En el sentido de dar existencia a la fracción anterior como línea geometría, se puede concluir que cualquier expresión algebraica podría ser expresada a partir de sustituciones proporcionales. En estos apartados no se muestra ningún tipo de construcción geométrica, se enuncia que todas pueden ser construidas por medio de triángulos semejantes, incluyendo cualquier fracción. En el texto se propuso que las proporciones sean interpretadas como fracciones, aspecto que relacionó la operación con una intención numérica, más que con una relacional.

En esta edición se otorga otro papel a las expresiones algebraicas; ahora desde ellas es posible solucionar los problemas transformando las expresiones indeterminadas en determinadas, por medio del remplazo de las letras por números.

[...] y estos también deben entenderse como problemas indeterminados que solo podemos resolver mediante la construcción de una infinidad de problemas definidos; lo cual se hace dando sucesivamente diferentes valores finitos a todas las incógnitas de la ecuación, excepto una, que siempre se considera desconocida [...]. (Lamy, 1758, p. 537)

En el caso de las ecuaciones de segundo grado se construyen los valores de las raíces por medio de las construcciones que aparecen en la Geometría (Descartes, 1996).

$x= \pm \frac{1}{2} a \pm \sqrt{\left(\frac{1}{4} a a-b b\right)} ; x= \pm \frac{1}{2} a \pm \sqrt{\left(\frac{1}{4} a a+b b\right)}$ , según corresponda la ecuación. Se describe un análisis de la expresión $\sqrt{\frac{1}{4} a a \pm b b}$ para determinar los casos en que existen raíces. 


\section{Conclusiones}

Al comparar el tratamiento del método durante las ediciones de texto se encuentran dos tipos de enunciados para las reglas; unas más amplias, menos técnicas, menos matematizadas y más filosóficas, como las del primer y tercer grupo de ediciones. En el segundo grupo se encuentran reglas más algorítmicas, tratadas con un lenguaje más matemático, más técnico, menos filosófico.

Hubo una transformación durante las ediciones del papel de las imágenes en el texto. En la primera edición la reflexión sobre el diagrama producto de las condiciones del problema era fundamental para que el lector entendiera la importancia del método. Fundamentalmente, las preocupaciones estuvieron sobre la relación entre las magnitudes conocidas y desconocidas, su importancia en el montaje del diagrama y en la solución del problema. En las otras ediciones el diagrama tuvo poca reflexión, aunque en una gran parte de los problemas se usa. En algunos casos un mismo diagrama sirvió para solucionar más de un problema, se intentaba mostrar que un problema era parte de una situación que se podría generalizar.

En el caso de las construcciones para la solución de ecuaciones de primer y segundo grado, estas fueron desapareciendo a medida que las ecuaciones fueron clasificadas por la forma de los términos de la progresión que las solucionaba. En la última edición se evidencia el uso de fórmulas producto del uso algebraico de las proporciones, hay una modificación del método plateado por Descartes y se acerca a lo que hoy se considera es la actividad en la escuela.

El análisis del texto permite identificar que el método tuvo varias adaptaciones para convertirlo en un tema en el ámbito de enseñanza. Sus énfasis estuvieron marcados por la necesidad de solucionar problemas cada vez más generalizados, lo que implicó generar una dependencia cada vez más mayor de técnicas algebraicas.

\section{Agradecimientos}

El artículo es producto de las conversaciones con el profesor Gert Schubring, a quien agradezco su tiempo y los aportes realizados a este documento.

\section{Referencias}

Barbin, E. (2010). Evolving Geometric Proo$f_{s}$ in the Seventeenth Century: From Icons to Symbols. En H. Dans, $\mathrm{H}$. Jahnke y $\mathrm{H}$. Pulte (eds.), Explanation and Proof in Mathematics. Philosophical and Educational Perspectives (pp. 237-252). Springer. https://doi. org/10.1007/978-1-4419-0576-5 16

Belhoste, B. (1993). L'Enseignement des mathématiques dans les collèges oratoriens au XVIIle siècle. En Dans J. Ehrard (ed.), Le collège de Riom et l'enseignement oratorien en France au XVIIle siècle (pp. 141-160). CNRS-éditions, et Oxford, Voltaire foundation.

Bos, H. (2001). Redefinig Geometrical Exactness. Descartes' Transformation of the Early Modern Concept of Construction. Springer. https://doi. org/10.1007/978-1-4613-0087-8

Descartes, R. (1996). Discurso del método. La dióptrica. Los meteoros. La geometría (G. Quintás, trad.) Circulo de Lectores S. A.

Descartes, R. (2010). Reglas para la dirección del espíritu (J. Navarro Cordón, trad.). Alianza Editorial.

Lamy, B. (1685). Les éléments de Géométrie ou de la mesure du corps.

Lamy, B. (1692). Nouveaux éléments de Géométrie ou de la mesure du corps.

Lamy, B. (1710). Les éléments de Géométrie ou de la mesure de L'étendue.

Lamy, B. (1731). Les éléments de Géométrie ou de la mesure de L'étendue.

Lamy, B. (1734). Les éléments de Géométrie ou de la mesure de L'étendue.

Lamy, B. (1740). Les éléments de Géométrie ou de la mesure de L'étendue. 
Lamy, B. (1758). Les éléments de Géométrie ou de la mesure de L'étendue..

Macbeth, D. (2004). Viete, Descartes and the emergence of Modern Mathematics. Graduate Faculty Phisolophy Journal, 25(2), 87-117. https://doi.org/10.5840/gfpj200425212

Schubring, G. (1987). On the methodology of Analysing Historical Textbook: Lacroix as Textbook Autor. For the Learning of Mathematics, 7(3), 41-51.
Schubring, G. (2005). Conflicts between generalization, rigor, and intuition. Springer.

Stacey, K., Chick, H., Kendal, M. (eds.) (2004). The Future of the Teaching and Learning of Algebra. The 12th ICMI Study. Kluwer Academic Publishers. https://doi.org/10.1007/1-4020-8131-6

Sutherland, R., Rojano, T., Bell, A., Lins, R. (eds.) (2002). Perspectives on School Algebra. Kluwer Academic Publishers. https://doi. org/10.1007/0-306-47223-6 